Sociohistórica

ISSN: 1852-1606

publicaciones@fahce.unlp.edu.ar

Universidad Nacional de La Plata

Argentina

\title{
La persistencia de las desigualdades en América Latina: desafíos para el siglo XXI, entrevista al antropólogo Luis Reygadas
}

Roberti, Eugenia

La persistencia de las desigualdades en América Latina: desafíos para el siglo XXI, entrevista al antropólogo Luis Reygadas

Sociohistórica, núm. 46, 2020

Universidad Nacional de La Plata, Argentina

DOI: https://doi.org/10.24215/18521606e115

Atribución no comercial compartir igual (CC BY-NC-SA) 4.0 


\title{
La persistencia de las desigualdades en América Latina: desafíos para el siglo XXI, entrevista al antropólogo Luis Reygadas
}

\author{
Eugenia Roberti \\ CONICET-Universidad Nacional Arturo Jauretche/ \\ Instituto de Desarrollo Económico y Social-Programa de \\ Estudios sobre Juventud, Educación y Trabajo/Facultad \\ de Humanidades y Ciencias de la Educación-Universidad \\ Nacional de La Plata, Argentina \\ mariaeugeniaroberti@gmail.com
}

DOI: https://doi.org/10.24215/18521606e115

Luis Reygadas es doctor en Ciencias Antropológicas y profesor-investigador del Departamento de Antropología por la Universidad Autónoma Metropolitana de Iztapalapa, México. En esta entrevista reconstruimos su itinerario y los núcleos temáticos de sus principales obras con la finalidad de analizar la persistencia de las desigualdades en América Latina. En particular, desandamos las preguntas originarias que motivaron su libro La apropiación, sus principales influencias, su énfasis en las especificidades de la región más desigual del mundo y los desafíos que enfrenta un estudio multidimensional de las desigualdades en el siglo actual. Todos estos temas no solo son abordados en profundidad, sino también interpelados a la luz del proceso de globalización, el lugar que ocupa el trabajo y sus nuevas formas de organización, la importancia de las políticas de igualación y el rol del Estado en la lucha contra la desigualdad.

- Fuiste pionero en adscribir a una perspectiva que atendiera el estudio de las desigualdades en América Latina; en este sentido, la primera pregunta es: ¿cómo fue el camino recorrido para llegar hasta este enfoque? Como en todo proceso investigativo, imagino que quedaron atrás posicionamientos, controversias con distintas tradiciones y conceptos que, incluso, pueden aparecer como intercambiables sin un adecuado sustento teórico. Me refiero a los conceptos de pobreza, vulnerabilidad o exclusión, los cuales no solo se insertan en tradiciones socioantropológicas específicas, sino que también están anclados en perspectivas teóricas e ideológicas que, como vos bien decís en uno de tus últimos artículos, también son políticos; la desigualdad siempre es política.

En este sentido, desde fines de los años ochenta, el neoliberalismo viene propugnando combatir la pobreza, posicionamiento que tiende a focalizar la problemática y a ocultarse tras una mirada meramente técnica, que desatiende el carácter relacional y político de esta problemática, y que en términos de políticas sociales latinoamericanas, específicamente en México y en Argentina, buscan la inclusión social a través de una "igualdad de oportunidades". Los trabajos de François Dubet (2014), bien señalan que este posicionamiento se esconde detrás un sesgo meritocrático.

Retomando la pregunta desde un marco regional, ecómo fue tu recorrido en términos de acoger un conjunto tradiciones, discutir con ciertos conceptos y perspectivas, y aprehender la potencialidad de adscribir a una aproximación de las desigualdades en términos analíticos?

- Comencé a analizar el tema de la desigualdad en el año 2003, por dos motivos: una incomodidad y una intuición. La incomodidad era que nadie hablaba sobre la desigualdad en esos años. Todos los noventa se hablaba sobre la globalización, la necesidad de reducir la pobreza, pero no se estaba escribiendo sobre desigualdad, que había sido un tema que se trabajó mucho en los años setenta, cuando yo estudié antropología.

Era una época en que el marxismo estaba muy fuerte en América Latina, en las escuelas de antropología y en muchas otras. En aquellos tiempos, la desigualdad sí era un tema, pero se estudiaba en términos muy 
estructurales y económicos. La idea en los setenta era: la desigualdad la produce el capitalismo, entonces, va a haber desigualdad hasta que no se elimine el capitalismo. Había un programa político, se la quería reducir, pero no se analizaba porque era un subproducto de la estructura general. Por el lado de la sociología funcionalista, se analizaba en los años sesenta en términos meritocráticos -qué hace cada quien, qué tiene cada quien-, atendiendo posiciones individuales en una estructura social.

Luego, se abandonó o se redujo muchísimo. Desde allí, emerge el tema de la pobreza como muy importante, pero me parecía que el análisis estaba cojo, al solo analizar la pobreza y no analizar la riqueza al mismo tiempo. También, se hablaba del concepto de exclusión, que le veía un potencial, en el sentido de una crítica a una sociedad que los había excluido, pero este concepto tenía cierto resabio funcionalista de la teoría de la marginalidad, que decía que los marginados estaban mal porque estaban fuera del sistema, el sistema funcionaba bien y ellos estaban fuera.

Hay cosas muy interesantes en los estudios sobre pobreza y sobre exclusión, pero no estaban captando esa parte de qué relación hay entre incluidos y excluidos, entre pobres y ricos. Entonces era una molestia, una incomodidad, porque yo lo que había estudiado desde finales de los setenta era sobre todo el trabajo industrial, sus procesos de trabajo y de organización sindical. Un poco había trabajado algo sobre desigualdad, pero no era mi tema, entonces era una molestia y una intuición. Mi intuición era que la desigualdad aumentaba y no la estábamos estudiando: con la globalización aparecieron fortunas enormes, empresas que hacían negocios en gran escala, y por el neoliberalismo se había perdido mucho de las políticas redistributivas.

Por otro lado, durante la década de los noventa en la antropología mexicana se había estudiado mucho el tema de cómo estaba cambiando la cultura con la globalización. El tema de la cultura había sido muy importante en esa década, y me había interesado en estudiarlo. Mi libro titulado Ensamblando culturas analiza los procesos de trabajo en la industria maquiladora, en el norte de México y en Guatemala, desde un punto de vista cultural. Entonces, quería reunir esa preocupación respecto a cómo la cultura influía en la desigualdad. En mi formación, más de tipo marxista, la cultura no ocupaba un lugar importante, la desigualdad la producía la propiedad o no propiedad de los medios de producción. Ya a esas alturas este enfoque me parecía muy limitado; sostenía que era importante estudiar los procesos culturales asociados a la desigualdad, también eso era parte del recorrido.

En un principio, las preguntas iniciales fueron ¿qué está pasando con la desigualdad en la época de la globalización? Había opiniones encontradas, por ejemplo, la incorporación de China a la producción de mercado sacó a cientos de millones de la pobreza; esto hizo que disminuyera la desigualdad mundial. Otro ejemplo era India. Sin embargo, al mismo tiempo había aumentado la desigualdad en otros países y a su interior. La literatura no era clara respecto a esta relación entre globalización y desigualdad; había tendencias y contratendencias.

La otra pregunta era qué papel jugaba la política y la cultura en la producción de las desigualdades. Resistía a quedarme con aquella respuesta simple que remitía solo a factores económicos y, particularmente, a aquello que había destacado la tradición crítica: la propiedad. Había leído a Max Weber y Pierre Bourdieu: la desigualdad también era producida por el estilo de vida, las formas de consumo, el modo de vida, entre otra serie de cosas. Esas fueron las dos preocupaciones: globalización y desigualdad/ cultura y desigualdad, concibiendo a la desigualdad como una relación de poder.

Y el camino a veces también lo fijan las condiciones institucionales. Gané una convocatoria de la Universidad Estatal de Nueva York en Stony Brook, para estudiar la desigualdad social en América Latina desde un punto de vista histórico-político-cultural. Allí, se incorpora la tercera pregunta: América Latina era como el mejor lugar para estudiar la desigualdad. Ya no solo era cómo influía la cultura y el poder en la desigualdad, qué pasaba con la desigualdad en la globalización, sino también por qué Latinoamérica era la región más desigual. Esos son los tres temas que trato en el libro La apropiación: Destejiendo las redes de la desigualdad, publicado en el año 2008. 
En el tema cultura y desigualdad, dos autores fueron claves. Por un lado, Michèle Lamont (1992), que había estudiado cómo las fronteras simbólicas entre diversos grupos sociales producen desigualdades. Esta autora pone en el centro del estudio sobre la desigualdad el plano de lo cultural, y, más específicamente, lo simbólico; idea que ya estaba presente en las obras de Max Weber y Pierre Bourdieu. Sin embargo, no estaba claro el tema de qué relación tiene esto con lo económico, y quien me ayuda a entenderlo es Charles Tilly (2000). Si hay una influencia fuerte en mi libro es Tilly, dado que relaciona las categorías/ clasificaciones con la distribución de recursos.

Siguiendo a este autor, se crean categorías, por ejemplo, hombre-mujer, negro-blanco, etc. -dimensión cultural-, que se asocian con una jerarquía -dimensión política-. Esta jerarquía establece quién está arriba, quién abajo; quién está adentro, quién afuera, lo cual se vincula luego con el flujo de recursos dimensión económica-. Esa idea que está en Tilly; es el hilo conductor de mi libro: cómo la desigualdad es multidimensional, al ser producida por factores culturales: cómo clasificamos a la gente, cómo la categorizamos, qué fronteras simbólicas creamos entre los grupos; factores políticos: cómo se transforma en jerarquías, relaciones asimétricas de poder, y, factores económicos: cómo se produce que algunos detenten ventajas y otros desventajas, a unos les toque más y a otros menos.

El libro trata de abordar esos múltiples factores desde una mirada teórica y de trazar un puente entre los estudios de estratificación social, centrados en el individuo, en donde la desigualdad se aborda en función de los activos o capitales con que cuenta el individuo, qué características tienen sus padres o qué habían estudiado, y los estudios estructurales, donde el problema radica en cómo está organizada la estructura social. El supuesto detrás residía en que si transformamos la estructura pero no transformamos a los individuos y sus circunstancias concretas, la desigualdad se reproduce. Una clave de lectura para mí fue Bourdieu, con su teoría del habitus, al explicar cómo la estructura social se personaliza, se vuelve individuo.

Mi reflexión giraba también con lo que había pasado en los países socialistas, donde se transformó la estructura social, los salarios y ciertas oportunidades educativas se volvieron igualitarios, pero algunos patrones culturales no habían cambiado. Es decir, el habitus de las clases medias y altas en Cuba seguía siendo similar al de sus padres y abuelos, mientras que el habitus de los sectores más pobres (digo Cuba, pero podría ser también el caso de Polonia, Rusia o Ucrania) no había cambiado tanto. Pese a ser sociedades muy igualitarias, las oportunidades eran diferentes para los diferentes sectores sociales.

En cuanto al recorrido entre globalización y desigualdad, allí encontré estudios que mostraban que en algunos países la desigualdad había aumentado con la globalización, y estudios que señalaban, por el contrario, que se había reducido. No es una relación tan simple, depende en parte de cómo se insertaron los países a la globalización. Algunos países asiáticos primero impulsaron políticas igualitarias a partir de desarrollar, sobre todo, un sistema educativo y sistema tecnológico fuerte. Es el caso de Japón, primero, y luego de Corea del Sur, Singapur, los tigres asiáticos. En estos países, muchos sectores de la población se pudieron incorporar a producir para la globalización y sacar beneficios.

Mientras que en países muy desiguales, por ejemplo, de América Latina, se entró a la globalización sin haber igualado antes. De allí que se benefician de la globalización solo algunas empresas, que pueden producir para la exportación o dedicarse a importar, y los sectores profesionistas que trabajan en compañías transnacionales o que iniciaban nuevos negocios con la globalización. En contraposición, aquellos sectores que no estaban preparados para competir en el mercado mundial, se vieron rezagados. Así, en esos casos donde la globalización se asocia a políticas neoliberales, se produce una mayor desigualdad. En última instancia, el problema no era la globalización, sino cómo se insertaban y qué configuraciones institucionales tenían los distintos países.

Por último, el tema de por qué América Latina es la región más desigual se explica por múltiples factores. La bibliografía es más consistente sobre este punto. La importancia del pasado colonial establece una matriz social en muchos países de América Latina, donde existe una minoría de origen europeo con altos ingresos, que se forma en la colonia pero que se reproduce luego en los últimos doscientos años, mientras que los 
sectores indígenas, mestizos, negros, mulatos, afrodescendientes no tienen las mismas oportunidades frente a la concentración de la tierra y la debilidad del Estado.

Por esta razón, la clave para entender qué países son más o menos desiguales, radica en las políticas para reducir la desigualdad. La distribución primaria en América Latina no es tan diferente a la de Europa. En muchos países europeos, sobre todo los del norte de Europa, Alemania, los países escandinavos, lo que adquiere importancia es la redistribución secundaria; el Estado redistribuye salud, educación e ingresos. Entonces, la manera en que funciona el Estado llamado social o de bienestar es la clave para entender los alcances de la lucha contra la desigualdad.

Mi hipótesis, que aparece en el libro como provocación, es que la desigualdad contemporánea tiene ver con que tenemos una economía globalizada (con precios y mercados financieros globalizados), pero no tenemos instancias globales para reducir la desigualdad. Cuando la economía era local, campesina, la desigualdad se producía en ese nivel y se contaba con mecanismos locales para contrarrestarla. En México, el ejemplo clásico eran las fiestas del pueblo: a quienes les fue mejor en la cosecha, pagan la fiesta. Frente a un Estado nacional, la desigualdad ya no es a nivel local, y es por medio de impuestos progresivos que se redistribuye la riqueza.

Ahora bien, en los últimos cuarenta años estamos frente a una economía que es global, pero carecemos de mecanismos globales para redistribuir la desigualdad. Las empresas que más ganan mandan su dinero a paraísos fiscales o no pagan los impuestos suficientes en muchos de los países de donde obtienen su riqueza. Así, las empresas transnacionales generan ganancias impresionantes y sus ejecutivos tienen supersalarios, tal como analiza Thomas Piketty: un directivo de una empresa transnacional puede ganar lo mismo que tres mil trabajadores de esa empresa juntos, sin cobrársele impuestos proporcionales a ese tamaño de la riqueza.

Mi conclusión es pesimista, respecto a que la desigualdad se agrava en esta época; y a la vez optimista, en razón de que la alternativa sería crear mecanismos transnacionales de redistribución de la riqueza y beneficios. En ese sentido, acuerdo con Piketty respecto a la necesidad de generar un impuesto mundial al capital. Sin embargo, desacuerdo en que ese impuesto deba usarse para la renta básica universal. Si bien contribuiría a la redistribución de la riqueza, se genera una nueva clasificación: los que aportan, pagan impuestos, y los que reciben beneficios.

En última instancia, aunque es interesante la propuesta de la renta básica universal, vuelve a dividir a la sociedad entre beneficiados de las políticas públicas y trabajadores que pagan impuestos. Se crea una polarización, que es la que estamos viendo en muchos países de América Latina, donde, si bien no hay ingreso básico universal, sí hubo en los últimos años gobiernos que trataban de reducir la desigualdad por la vía de los subsidios y las transferencias monetarias condicionadas o no condicionadas, creándose así un nuevo conflicto estructural.

- En este marco, ¿qué particularidades tiene focalizar el estudio sobre las desigualdades en América Latina? En tanto región más desigual del mundo, qué recaudos habría que tomar para su análisis. Y en particular, como especialista dentro del campo de la antropología del trabajo, ¿qué lugar tiene el trabajo hoy en día en una sociedad tendiente hacia la precarización, flexibilización e inmaterialidad? ¿Cómo (re)pensar el vínculo entre la (des)igualdad y las nuevas formas de organización del trabajo?

- Respecto a la primera pregunta, dos cuestiones destacan sobre las ventajas de focalizarse en América Latina. En primer lugar, con el descubrimiento de América, América Latina se vuelve la bisagra entre un mundo tradicional (local, preindustrial, campesino) y un mundo que comienza a globalizarse. América Latina se vuelve una sociedad en dos velocidades: por un lado, algunos sectores de la economía de los países coloniales estaban supermodernos vendiendo para el mercado, con una producción muy avanzada y global para el momento; es el ejemplo de las plantaciones de caña y los ingenios azucareros; por otro lado, grandes regiones de Latinoamérica estaban enfocadas hacia el autoconsumo y la producción de subsistencia.

América Latina a partir del período colonial encarna la unión entre lo local y lo global, entre lo moderno y lo tradicional. De allí que, desde la CEPAL se haga referencia a las asimetrías estructurales de las economías latinoamericanas. En consecuencia, considero que la clave para entender la desigualdad no es la pobreza, sino 
la riqueza. De lo contrario, ¿por qué no es la región más desigual el sudeste asiático o el áfrica subsahariana? El problema como ocurre en América Latina es que se combina mucha riqueza con mucha pobreza. La desigualdad aumenta con la aparición de nuevos métodos productivos y formas novedosas de organización de la economía que generan mucha riqueza. América Latina, en ese sentido, explica mucho de la desigualdad contemporánea, porque es donde primero se vio esa combinación de una economía globalizada, muy dinámica, con economías locales muy tradicionales, de bajo nivel de productividad.

Un segundo elemento es que en América Latina se da una yuxtaposición entre factores étnico-raciales y económicos. Donde hay mayor desigualdad en el mundo es en América Latina y en algunos países africanos que tienen alguna característica similar. Por ejemplo, Sudáfrica, donde existe una elite blanca, es muy desigual. Al observar los coeficientes de Gini (desigualdades de ingresos) de esos países, se parecen a América Latina: existe una minoría de origen europeo con altos niveles educativos, que participan en un sector de la economía dinámico, con una mayoría que no. La sobreposición de las categorías étnicas con las categorías de clase es lo que explica la desigualdad persistente.

En el caso de América Latina, ya no es suficiente para entender las desigualdades contemporáneas la cuestión de las superfortunas del uno por ciento más rico, las cuales se explican por la economía global y la falta de regulación del capital financiero. Ahí no bastan las políticas de acción afirmativa y la búsqueda por lograr la equidad para los grupos indígenas y afrodescendientes. Hay que acudir al viejo Marx, Hilferding y Piketty, quien explica estas superganancias a nivel global, y que requieren de otra política, que va más allá de la acción afirmativa, de la creación de oportunidades para sectores en desventaja, y que tiene que ver con cómo gravar las grandes fortunas.

Respecto a la segunda pregunta vinculada con el tema del trabajo, a mí me parece fundamental. Como bien muestra Piketty, existen dos desigualdades: la desigualdad de capital, que es la más fuerte, y la desigualdad entre quienes trabajan, o entre quienes trabajan y quienes no trabajan. Considero que el principal igualador en una sociedad, desde la sociedad industrial, es el trabajo; no solo porque se ha asociado al mismo la seguridad social, sino porque es la principal fuente de ingresos.

Si queremos reducir la desigualdad, la mejor vía sería que la mayor parte de la población tuviera un empleo digno. Esa es mi discusión con los defensores del ingreso ciudadano, de la renta universal básica. No estoy en contra de ella, pero creo que el reto es cómo propiciar un acceso al trabajo para más personas. Además, porque el trabajo da derechos, identidad, redes sociales, posibilidad de organizarse, dignifica, entre otras cosas.

Por esta razón, no estoy en contra de que haya todo tipo de apoyos, rentas básicas, subsidios, etc. a quienes más lo necesiten, pero creo que la clave está en redistribuir el trabajo digno. Las sociedades modernas más igualitarias, si hablamos de la Revolución Industrial para acá, han sido los países socialistas y los países capitalistas que estaban cercanos al pleno empleo; allí las desigualdades de ingreso disminuyeron.

Ahora bien, acuerdo en desvincular servicios de salud del empleo; creo que sí es obligación del Estado proporcionar salud pública de calidad a toda la población, independientemente de que esté empleada o no. Pero eso no debe impedir que se busque lograr igualdad de oportunidades en el acceso al trabajo. La propuesta más igualitaria, según mi postura, no es el ingreso básico universal, sino la disminución radical de la jornada de trabajo: que todos trabajen cuatro horas en un empleo digno, con acceso a la seguridad social; no que algunos trabajen ocho horas y ganen mucho, mientras que otros tengan un empleo precario o una ayuda social y perciban la tercera parte.

Para mí es fundamental el tema del trabajo, porque el trabajo ha sido el acceso a la ciudadanía, entre muchas otras cosas, y considero que no hay que dar por perdida esa batalla.

- El problema que yo veo ahí es que, justamente, las tendencias del mercado de trabajo son hacia la mayor desprotección y flexibilización. A esto se suma la dificultad de regular las nuevas plataformas digitales, donde uno se puede autoemplear (por nombrar algunos ejemplos, tales como Rappi, Airbnb y Uber). Es decir, eno se hace más difícil llegar a sistemas regulatorios en estas nuevas formas de organización del trabajo? Si bien, acuerdo con los significados que adquiere el trabajo, ligados a 
cuestiones identitarias, de organización social, de regulación del tiempo y de la vida, incluso, como hecho social en sí mismo; no podemos dejar de preguntarnos en qué condiciones el trabajo continúa siendo un pilar fundamental de integración.

- Bueno, ahora estoy estudiando el tema de las plataformas. Ahí también hay una oportunidad, es muy fácil regular ese trabajo. El trabajo en negro de alguien que vende piratería es muy difícil de regular, dado que está en una condición ilegal. En cambio, todas las actividades de las plataformas están registradas. Estamos a un click de que a todas esas actividades que hacen los rapitenderos, los conductores de Uber, los trabajadores de microtareas se les ponga un impuesto que paguen los patrones o alguna parte de los trabajadores, para su jubilación. Es decir, técnicamente es muy fácil cobrarle impuestos a las empresas que trabajan en plataforma, porque no están escondidas. Está registrado en una base de datos quiénes son, dónde están, cuántas horas trabajan y cuáles son sus ganancias. No es economía subterránea, claro que se necesitan condiciones y voluntad política para eso, pero las condiciones técnicas para dignificar ese trabajo existen. En el caso de Argentina, la plataforma Zolvers para empleadas domésticas ha ayudado a dignificar ese trabajo y a regularlo. El futuro radica, entonces, en cómo dignificar y regular esas nuevas formas de trabajo.

\section{- ¿Qué opinión tenés sobre el fenómeno de coworking?}

Lo analicé en algún texto. Ese es un negocio inmobiliario, se comparte un espacio de trabajo, pero en el fondo es un negocio rentista. Se presenta como una avanzada de un capitalismo innovador, así lo hizo The Hub, una de las empresas de coworking, pero en el fondo es un piso de doscientos metros cuadrados, que, en lugar de rentárselo a una sola persona, se lo rento a quinientos y obtengo más ganancia. Es un negocio inmobiliario, aunque se presenta como vanguardia, economía colaborativa, compartir espacios, etc.

Piketty analiza en profundidad el tema de la renta. En la época de David Ricardo, a principios del siglo XIX, se veía como un gran obstáculo para el desarrollo del capitalismo a los terratenientes agrícolas. Con la industrialización, la tierra como recurso productivo perdió importancia frente a la tecnología y las maquinarias. Sin embargo, en las actuales ciudades de millones de habitantes, la tierra posee un gran valor. Aquí los nuevos terratenientes son terratenientes urbanos, que obtienen una ventaja impresionante. Por esta razón, Piketty insiste en la necesidad de gravar al capital, a la propiedad de la tierra y a la herencia de los inmuebles. No solo para crear condiciones de igualación, sino también para promover el crecimiento económico.

En última instancia, el trabajo es fundamental para la igualdad y, como corolario, es fundamental la educación, para que la gente pueda acceder a buenos trabajos. Una de las limitaciones de la izquierda, en cuanto a sus políticas de igualación, es que no ha llevado a la práctica aquellas políticas públicas que se derivan de la obra de Bourdieu. A la hora de luchar contra la desigualdad, se pide que haya igualdad en la educación terciaria. Lo que Bourdieu demuestra es que el capital cultural y simbólico se forma en los primeros años de vida. Si queremos igualar, hay que igualar en los primeros años de vida, sale mucho más barato y tiene mucho más sentido igualar en la educación inicial -que es cuando se forma lo central del habitus-, que luchar porque haya igualdad en el grado o posgrado. La batalla cognitiva por formar las capacidades más importantes se da en los primeros años de vida. Está bien la gratuidad universitaria y que en esos niveles haya oportunidades para todos, pero las batallas más importantes son necesarias darlas con anterioridad, para llegar al mercado de trabajo en condiciones más parejas.

- Para ir terminando, hoy en día existe un consenso a nivel teórico en torno a pensar las desigualdades sociales en términos procesuales, multidimensionales y relacionales; sin embargo, las investigaciones empíricas la mayoría de las veces no permiten vislumbrar la conjunción de estos múltiples factores y niveles en sus abordajes. En este sentido, ¿cuáles creés que son los desafíos pendientes y qué avances recientes se observan en los estudios sobre las desigualdades?

- Yo creo que los enfoques procesuales sí abren espacio a la investigación empírica. Una de las tesis centrales es que la desigualdad no está hecha de una vez y para siempre, sino que se construye. Por lo tanto, se puede ir a investigar si en ciertas circunstancias, en determinada región y período, aumentó, disminuyó o cambió 
de tipo. Se abre un programa de investigación, donde el enfoque estructural solo permitía hacer agregados de grandes números, el enfoque procesual invita al estudio de caso, la etnografía, al estudio microsocial.

Lo que es muy difícil es desarrollar a nivel empírico una perspectiva multidimensional. El reto está en poder hacer, desde un punto de vista cualitativo, lo que hacen los estudios cuantitativos multifactoriales. Desde este enfoque se analiza, a través de una serie de procedimientos estadísticos, qué peso tiene un determinado factor en la producción de desigualdades, sea la escolaridad de los padres, el lugar de nacimiento, los años de escolaridad, entre otros. El reto es poder hacer estudios en los que incidan diferentes factores, pero desde abordajes cualitativos.

El desafío está, por un lado, en intentar llevar el tema multidimensional del discurso al análisis empírico, analizando qué tanto pesa el género, qué tanto pesa la edad, qué tanto pesa la clase social, etc.; y, por otro lado, es también observar los cambios en el tiempo. Por ejemplo, analizar qué ha pasado con la desigualdad en América Latina en los últimos años de este siglo, donde hubo tantas políticas para tratar de reducirla; en particular, en qué medida redujo la desigualdad el paso de un enfoque de transferencias condicionadas a un enfoque de derechos.

No tengo respuestas a estas preguntas, porque no he hecho ningún estudio empírico al respecto. Sin embargo, algo interesante que se encontró en un estudio reciente que realicé, sobre la situación del mercado laboral de la antropología en México, fue que el factor que más pasaba en la desigualdad de ingreso y de condiciones laborales de los antropólogos era la edad y el momento en que se estudió (Reygadas, 2019). Se encontraron desigualdades que tenían que ver con el género o la clase social, pero la gran desigualdad fue entre viejos y jóvenes. ¿Por qué?, porque los antropólogos mayores de cincuenta años, en el caso de México, entraban al mercado de trabajo cuando había pocos antropólogos y había muchas oportunidades; en cambio, los más jóvenes entraron a un mercado saturado. Aquí es el factor estructural ligado al mercado trabajo el que está creando las mayores desigualdades.

- Estaba pensando en qué medida juega un efecto cohorte, en función del momento en que ingresan al mercado laboral; o si se debe a un asunto de madurez ocupacional. Me pregunto qué pasará con esos jóvenes en la edad adulta.

- No hay datos precisos, pero sí resulta que las cohortes más jóvenes empiezan en una situación más desventajosa que las otras. Sí se observa ese efecto, en el sentido de que los más jóvenes entraron al mercado laboral ya en desventaja, comparando a cómo estaban al momento de ingreso las diferentes cohortes.

Este tema de lo estructural es importante. Si en los años setenta la tendencia era achacarle todo a la estructura, sin ver los factores étnicos y de género, luego se le da más importancia a estos factores, al demostrarse cómo el género y lo étnico produjeron grandes desigualdades en América Latina. Ahora bien, darle importancia a estos factores ha tenido también un costo: el pensar que el principal problema de la desigualdad está en la mentalidad de las personas. Es decir, que los principales obstáculos a la igualdad son el racismo y la mentalidad patriarcal. Son dos grandes obstáculos a la igualdad, pero no son los únicos, hay temas estructurales que no los resuelve un cambio de mentalidad.

La desigualdad de oportunidades que hay entre vivir en el Conurbano de Buenos Aires, la Ciudad de México o de Sao Pablo, con respecto a vivir en Chiapas, el norte de Argentina o el nordeste brasileño, son enormes; o vivir en Bogotá con respecto a vivir en el pacífico colombiano, donde hay muy pocas oportunidades. Allí se mezcla, porque el pacífico colombiano es donde vive la mayor parte de los negros y en el sur de México es donde vive una gran parte de la población indígena. Sin embargo, no se trata de una acción racista directa, sino de las consecuencias estructurales del racismo y del sexismo, que van dejando huella en el territorio y que van dejando huella en las estructuras educativas.

Para finalizar, sostengo que hay que seguir combatiendo las ideas racistas y los prejuicios machistas, por supuesto, pero también hay temas estructurales que hay que atacar; no basta con cambiar la mentalidad. Mientras no transformemos los flujos de recursos y las estructuras, será limitado el alcance de las políticas de inclusión étnica y de igualdad de género. 
Eugenia Roberti. La persistencia de las desigualdades en América Latina: desafíos Para el siglo XX...

\section{REFERENCIAS}

Dubet, F. (2014). Repensar la justicia social. Argentina: Siglo XXI Editores.

Lamont, M. (1992). The Culture of the French and the American Upper-Middle Class. Morality and Society series. Chicago: University of Chicago Press.

Reygadas, L. (2002). Ensamblando culturas. Diversidady conflicto en la globalización de la industria. Barcelona: Gedisa. Reygadas, L. (2008). La apropiación. Destejiendo las redes de la desigualdad. Barcelona: Anthropos/UAM-I.

Reygadas, L. (2019).Antropólog@s del milenio.Desigualdad, precarización y heterogeneidad en las condiciones laborales de la antropología en México. México: UAM/INAH/CIESAS/UIA/CEAS.

Tilly, C. (2000). La desigualdad persistente. Buenos Aires: Manantial.

\section{BY-NC-SA}

